\title{
UNIVERSALITY OF METHODS APPROXIMATING THE DERIVATIVE
}

\section{Gerd Herzog and Roland Lemmert}

We prove the existence of universal functions for mappings $T_{n}: C([0,1]) \rightarrow L^{p}([0,1])$, $0<p<1$, with $T_{n}(f) \rightarrow f^{\prime}(n \rightarrow \infty)$ on certain subsets of $C^{1}([0,1])$. As an application we conclude that there are continuous functions $f \in C([0,1])$, such that the derivatives of the Bernstein polynomials

$$
\left\{\left(B_{n}(f)\right)^{\prime}: n \in \mathbb{N}\right\}
$$

form a dense subset of $L^{p}([0,1])$ for each $0<p<1$.

\section{INTRODUCTION}

Let $C([0,1])$ denote the Banach space of continuous functions $f:[0,1] \rightarrow \mathbb{R}$ endowed with the maximum norm $\|\cdot\|$, let $C^{1}([0,1])$ denote the normed space of continuously differentiable functions endowed with the norm

$$
\|f\|_{1,1}=\|f\|+\int_{0}^{1}\left|f^{\prime}(t)\right| d t
$$

and for $0<p<1$ let $L^{p}([0,1])$ denote the $F$-space of all measurable functions $g:[0,1]$ $\rightarrow \mathbb{R}$ with

$$
\int_{0}^{1}|g(x)|^{p} d x<\infty
$$

(modulo sets of Lebesgue measure zero), endowed with the metric

$$
d\left(g_{1}, g_{2}\right)=\int_{0}^{1}\left|g_{1}(x)-g_{2}(x)\right|^{p} d x .
$$

Let $T_{n}, n \in \mathbb{N}$ be a family of mappings

$$
T_{n}: C([0,1]) \rightarrow L^{p}([0,1]) .
$$

We shall give conditions on the mappings $T_{n}, n \in \mathbb{N}$, satisfied by several classical approximation methods, such that this family of mappings has universal elements.

Received 16th January, 2006

Copyright Clearance Centre, Inc. Serial-fee code: 0004-9727/06 \$A2.00+0.00. 


\section{A UNIVERSALITÝ THEOREM}

TheOREM 1. Let $0<p<1$, and let the mappings

$$
T_{n}: C([0,1]) \rightarrow L^{p}([0,1]), \quad n \in \mathbb{N}
$$

have the following properties:

1. Each mapping

$$
T_{n}: C([0,1]) \rightarrow L^{p}([0,1]), \quad n \in \mathbb{N}
$$

is continuous;

2. There is a dense subset $S$ of

$$
\left(C^{1}([0,1]),\|\cdot\|_{1,1}\right)
$$

such that $T_{n}(f) \rightarrow f^{\prime}(n \rightarrow \infty)$ for each $f \in S$.

Then the set of functions $f \in C([0,1])$ such that

$$
\left\{T_{n}(f): n \in \mathbb{N}\right\} \text { is dense in } L^{p}([0,1])
$$

is a dense $G_{\delta}$ subset of $C([0,1])$.

REMARKs. 1. If $0<p_{1} \leqslant p_{2}<1$ then $L^{p_{2}}([0,1]) \subseteq L^{p_{1}}([0,1])$ and the embedding

$$
E: L^{p_{2}}([0,1]) \rightarrow L^{p_{1}}([0,1]),
$$

$E(g)=g$ is dense and continuous. Hence, a standard category argument proves that if the assumptions of Theorem 1 hold for each $0<p<1$, then the set of functions $f \in C([0,1])$ such that

$$
\left\{T_{n}(f): n \in \mathbb{N}\right\} \text { is dense in } L^{p}([0,1]) \text { for each } 0<p<1
$$

is a dense $G_{\delta}$ subset of $C([0,1])$.

2. As will be discussed in Section 6, Theorem 1 cannot be generalised to the case $p \geqslant 1$.

\section{UNIVERSAL ELEMENTS}

To prove Theorem 1 we shall make use of the Universality Criterion of GrosseErdmann [4, Theorem 1].

Suppose that $Y_{1}$ is a Baire space, $Y_{2}$ is second countable, and $T_{j}: Y_{1} \rightarrow Y_{2}(j \in J)$ is a family of continuous mappings. An element $y \in Y_{1}$ is called universal for this family if $\left\{T_{j} y: j \in J\right\}$ is dense in $Y_{2}$. Let $U$ denote the set of all universal elements.

Propos I TION 1. (Universality Criterion) Equivalent are:

1. The set $U$ is a dense $G_{\delta}$-subset of $Y_{1}$.

2. The set $U$ is dense in $Y_{1}$.

3. The set $\left\{\left(y, T_{j} y\right): y \in Y_{1}, j \in J\right\}$ is dense in $Y_{1} \times Y_{2}$. 
4. Dense subsets of $L^{p}([0,1])$.

First note that $C([0,1])$ is a dense subset of $L^{p}([0,1])$, see $[3]$. The following propositions prove that functions in $L^{p}([0,1])$ may be approximated by derivatives of uniformly bounded functions:

Proposition 2. Let $0<p<1$. Then

$$
D_{0, \varepsilon}:=\left\{w^{\prime}: w \in C^{1}([0,1]),\|w\| \leqslant \varepsilon\right\}
$$

is a dense subset of $L^{p}([0,1])$ for each $\varepsilon>0$.

Proof: Fix $\varepsilon>0$. It is sufficient to approximate continuous functions, so let $g \in C([0,1])$. Let $\varphi \in C^{\infty}(\mathbb{R},[0, \infty))$ satisfy $\operatorname{supp}(\varphi) \subseteq[0,1]$ and

$$
\int_{0}^{1} \varphi(x) d x=1
$$

Since $g$ is continuous we can choose $m \in \mathbb{N}$ such that $2\|g\| / m \leqslant \varepsilon$. Set

$$
\alpha_{k}=m \int_{k / m}^{(k+1) / m} g(s) d s \quad(k=0, \ldots, m-1) .
$$

We have

$$
\begin{aligned}
\beta_{k} & :=\int_{k / m}^{(k+1) / m}\left|g(t)-\alpha_{k} \varphi\left(m\left(t-\frac{k}{m}\right)\right)\right| d t \\
& \leqslant \frac{1}{m}\left(\|g\|+\left|\alpha_{k}\right|\right) \leqslant \frac{2\|g\|}{m} \leqslant \varepsilon \quad(k=0, \ldots, m-1) .
\end{aligned}
$$

Define $v, w:[0,1] \rightarrow \mathbb{R}$ by

$$
v(x)=-\alpha_{k} \varphi\left(m\left(x-\frac{k}{m}\right)\right)(x \in[k / m,(k+1) / m], \quad k=0, \ldots, m-1),
$$

and

$$
w(x)=\int_{0}^{x} g(t)+v(t) d t \quad(x \in[0,1]) .
$$

Note that $\operatorname{supp}(\varphi) \subseteq[0,1]$ implies that $v$ is continuous (even in $C^{\infty}$ ), hence $w \in C^{1}([0,1])$.

We have $w(k / m)=0(k=0, \ldots, m-1)$, since $w(0)=0$, and

$$
\begin{aligned}
w((k+1) / m)-w(k / m) & =\int_{k / m}^{(k+1) / m} g(t)+v(t) d t \\
& =\frac{\alpha_{k}}{m}-\frac{\alpha_{k}}{m} \int_{k / m}^{(k+1) / m} m \varphi\left(m\left(t-\frac{k}{m}\right)\right) d t=0,
\end{aligned}
$$


as

$$
\int_{k / m}^{(k+1) / m} m \varphi\left(m\left(t-\frac{k}{m}\right)\right) d t=1
$$

Let $x \in[k / m,(k+1) / m]$. Then, by the choice of $m$,

$$
|w(x)|=|w(x)-w(k / m)| \leqslant \int_{k / m}^{(k+1) / m}|g(t)+v(t)| d t=\beta_{k} \leqslant \varepsilon .
$$

Hence $w \in D_{0, \varepsilon}$ for each $\varphi$ with the chosen properties.

Next,

$$
\begin{aligned}
d\left(g, w^{\prime}\right) & =\int_{0}^{1}\left|g(t)-w^{\prime}(t)\right|^{p} d t \\
& =\int_{0}^{1}|v(t)|^{p} d t \\
& =\sum_{k=0}^{m-1}\left|\alpha_{k}\right|^{p} \int_{k / m}^{(k+1) / m}\left|\varphi\left(m\left(t-\frac{k}{m}\right)\right)\right|^{p} d t \\
& =\left(\sum_{k=0}^{m-1} \frac{\left|\alpha_{k}\right|^{p}}{m}\right) \int_{0}^{1}|\varphi(t)|^{p} d t \\
& =m^{p-1}\left(\sum_{k=0}^{m-1}\left|\int_{k / m}^{(k+1) / m} g(t) d t\right|^{p}\right) \int_{0}^{1}|\varphi(t)|^{p} d t=: c \int_{0}^{1}|\varphi(t)|^{p} d t
\end{aligned}
$$

and likewise these equations are valid for each $\varphi$ with the chosen properties.

Let $\delta>0$. Since $0<p<1$ we can choose $\varphi$ such that in addition

$$
d\left(g, w^{\prime}\right)=c \int_{0}^{1}|\varphi(t)|^{p} d t \leqslant \delta
$$

by choosing $\operatorname{supp}(\varphi)$ sufficiently small.

As a consequence of Proposition 2 we get

Proposition 3. Let $0<p<1$, and let $f \in C([0,1])$. Then

$$
D_{f, \varepsilon}:=\left\{w^{\prime}: w \in C^{1}([0,1]),\|w-f\| \leqslant \varepsilon\right\}
$$

is a dense subset of $L^{p}([0,1])$ for each $\varepsilon>0$.

Proof: Fix $\varepsilon>0$, let $g \in C([0,1])$, and let $\delta>0$. Since $C^{1}([0,1])$ is a dense subset of $C([0,1])$ we can choose $u \in C^{1}([0,1])$ such that $\|u-f\| \leqslant \varepsilon / 2$. According to Proposition 2 there is a function $v \in D_{0, \varepsilon / 2}$ such that

$$
d\left(v^{\prime}+u^{\prime}, g\right)=d\left(v^{\prime}, g-u^{\prime}\right) \leqslant \delta .
$$

Set $w=v+u$. We have $d\left(w^{\prime}, g\right) \leqslant \delta$, and

$$
\|w-f\| \leqslant\|w-u\|+\|u-f\|=\|v\|+\|u-f\| \leqslant \varepsilon
$$

that is $w \in D_{f, \varepsilon}$. 


\section{Proof of Theorem 1.}

We verify condition 3 of Proposition 1.

Each

$$
T_{n}: C([0,1]) \rightarrow L^{p}([0,1]), \quad n \in \mathbb{N}
$$

is continuous, $C([0,1])$ is a Baire space, and $L^{p}([0,1])$ is separable. Let $f \in C([0,1])$, let $g \in L^{p}([0,1])$ be without loss of generality in $C([0,1])$, and let $\varepsilon>0$. Condition 3 of Proposition 1 is verified if we can show that there is a function $q \in C([0,1])$ and a number $n_{0} \in \mathbb{N}$ such that

$$
\|q-f\| \leqslant \varepsilon \quad \text { and } \quad d\left(T_{n_{0}}(q), g\right) \leqslant \varepsilon .
$$

According to Proposition 3 there exists $w \in D_{f, \varepsilon / 2}$ such that $d\left(w^{\prime}, g\right) \leqslant \varepsilon / 3$. Next, since $S$ is dense in $\left(C^{1}([0,1]),\|\cdot\|_{1,1}\right)$ there exists $q \in S$ such that

$$
\|q-w\| \leqslant \frac{\varepsilon}{2} \quad \text { and } \quad d\left(q^{\prime}, w^{\prime}\right) \leqslant \frac{\varepsilon}{3},
$$

since convergence in $L^{1}([0,1])$ implies convergence in $L^{p}([0,1])$, compare [3, Lemma 1$]$.

In particular we have $\|q-f\| \leqslant\|q-w\|+\|w-f\| \leqslant \varepsilon$, and

$$
d\left(q^{\prime}, g\right) \leqslant d\left(q^{\prime}, w^{\prime}\right)+d\left(w^{\prime}, g\right) \leqslant \frac{2}{3} \varepsilon .
$$

Since $q \in S$ we have

$$
T_{n}(q) \rightarrow q^{\prime} \quad(n \rightarrow \infty) .
$$

Hence $d\left(T_{n_{0}}(q), q^{\prime}\right) \leqslant \varepsilon / 3$ for some $n_{0} \in \mathbb{N}$. Thus

$$
d\left(T_{n_{0}}(q), g\right) \leqslant d\left(T_{n_{0}}(q), q^{\prime}\right)+d\left(q^{\prime}, g\right) \leqslant \varepsilon .
$$

\section{Applications.}

1. Theorem 1 applies to the derivatives of Bernstein polynomials: Let

$$
\left(B_{n}(f)\right)(x)=\sum_{k=0}^{n}\left(\begin{array}{l}
n \\
k
\end{array}\right) f\left(\frac{k}{n}\right) x^{k}(1-x)^{n-k},
$$

and let $T_{n}: C([0,1]) \rightarrow L^{p}([0,1])$ be defined by $T_{n}(f)=\left(B_{n}(f)\right)^{\prime}$. Obviously each $T_{n}, n \in \mathbb{N}$ is continuous and condition 2. of Theorem 1 holds for $S=C^{1}([0,1])$, since $\left(B_{n}(f)\right)^{\prime} \rightarrow f^{\prime}(n \rightarrow \infty)$ even in $C([0,1])$ for each $f \in C^{1}([0,1])$, see [7, Section 1.8]. Thus, the set of continuous functions $f:[0,1] \rightarrow \mathbb{R}$ such that

$$
\left\{\left(B_{n}(f)\right)^{\prime}: n \in \mathbb{N}\right\} \text { is dense in } L^{p}([0,1]) \text { for each } 0<p<1
$$


is a dense $G_{\delta}$ subset of $C([0,1])$.

2. Theorem 1 applies to the derivatives of Lagrange interpolation polynomials: Let $L_{n}(f)$ denote the Lagrange interpolation polynomial of $f$ of degree at most $n$ with respect to arbitrary nodes

$$
0 \leqslant \xi_{0}^{(n)}<\xi_{1}^{(n)}<\cdots<\xi_{n}^{(n)} \leqslant 1 \quad(n \in \mathbb{N}),
$$

and let $T_{n}: C([0,1]) \rightarrow L^{p}([0,1])$ be defined by $T_{n}(f)=\left(L_{n}(f)\right)^{\prime}$.

Again, each $T_{n}, n \in \mathbb{N}$ is continuous and condition 2. of Theorem 1 holds for the set $S$ of all polynomials, since $\left(L_{n}(f)\right)^{\prime}=f^{\prime}$ if $f \in S$ and $n \geqslant \operatorname{deg} f$. Again, the set of continuous functions $f:[0,1] \rightarrow \mathbb{R}$ such that

$$
\left\{\left(L_{n}(f)\right)^{\prime}: n \in \mathbb{N}\right\} \text { is dense in } L^{p}([0,1]) \text { for each } 0<p<1
$$

is a dense $G_{\delta}$ subset of $C([0,1])$.

REMARK. For universal properties of the operators $L_{n}: C([0,1]) \rightarrow L^{p}([0,1])$ with $p \geqslant 1$ (which depend on the choice of the nodes) see [5].

3. Let $\left(\lambda_{n}\right)_{n=1}^{\infty}$ be a sequence with $\left|\lambda_{n}\right| \in(0,1]$ and with limit 0 . Theorem 1 applies to difference quotients: For $f \in C([0,1])$ let $f_{e}:[-1,2] \rightarrow \mathbb{R}$ be the extension defined by

$$
f_{\boldsymbol{e}}(x)=\left\{\begin{array}{cc}
2 f(1)-f(2-x) & (x \in(1,2]) \\
f(x) & (x \in[0,1]) \\
2 f(0)-f(-x) & (x \in[-1,0))
\end{array},\right.
$$

and let $T_{n}: C([0,1]) \rightarrow L^{p}([0,1])$ be defined by

$$
\left(T_{n}(f)\right)(x)=\frac{f_{e}\left(x+\lambda_{n}\right)-f_{e}(x)}{\lambda_{n}} .
$$

By standard reasoning each $T_{n}$ is continuous and $T_{n}(f) \rightarrow f^{\prime}$ in $C([0,1])$ for each $f \in S:=C^{1}([0,1])$. Once more, the set of continuous functions $f:[0,1] \rightarrow \mathbb{R}$ such that

$$
\left\{T_{n}(f): n \in \mathbb{N}\right\} \text { is dense in } L^{p}([0,1]) \text { for each } 0<p<1
$$

is a dense $G_{\delta}$ subset of $C([0,1])$.

This result is, in a certain sense, the one-dimensional case of Joó's generalisation ([6, Theorem I]) of Marcinkiewicz's classical result [8] on universal primitives. In [1] and [2] Bogmér, Sövegjártó and Buczolich proved that there is no universal primitive in $L^{1}([0,1])$ for $p \geqslant 1$. In particular if $p \geqslant 1$, then there is no $f \in C([0,1])$ such that

$$
\left\{T_{n}(f): n \in \mathbb{N}\right\} \text { is dense in } L^{p}([0,1]) .
$$




\section{REFERENCES}

[1] A. Bogmér and A. Sövegjártó, 'On universal functions', Acta Math. Hungar. 49 (1987), 237-239.

[2] Z. Buczolich, 'On universal functions and series', Acto Math. Hungar. 49 (1987), 403-414.

[3] M.M. Day, 'The spaces $L^{p}$ with $0<p<1$ ', Bull. Amer. math. Soc. 46 (1940), 816-823.

[4] K.-G. Grosse-Erdmann, 'Universal families and hypercyclic operators', Bull. Amer. Math. Soc. (N.S.) 36 (1999), 345-381.

[5] G. Herzog, 'On universal functions and interpolation', Analysis 11 (1991), 21-26.

[6] I. Joó, 'On the divergence of eigenfunction expansions', Ann. Univ. Sci. Budapest Eötvös Sect. Math. 32 (1989), 3-36.

[7] G.G. Lorentz, Bernstein polynomials, Mathematical Expositions 8 (University of Toronto Press X, Toronto, 1953).

[8] J. Marcinkiewicz, 'Sur les nombres dérivés.', Fund. Math. 24 (1935), 305-308.

\section{Mathematisches Institut I}

Universität Karlsruhe

D-76128 Karlsruhe

Germany

e-mail: Gerd.Herzog@math.uni-karlsruhe.de

Roland.Lemmert@math.uni-karlsruhe.de 